\title{
AN EXPRESSION FOR THE FIRST CONJUGATE POINT FOR AN $n$ TH ORDER NONLINEAR DIFFERENTIAL EQUATION
}

\author{
ALLAN C. PETERSON
}

\begin{abstract}
It is proved that the first conjugate point for an $n$th order nonlinear differential equation is the infimum of the first conjugate points of the corresponding variational equations.
\end{abstract}

We are concerned with the $n$th order nonlinear differential equation

$$
y^{(n)}=f\left(x, y, y^{\prime}, \ldots, y^{(n-1)}\right)
$$

where we assume throughout that:

(A) $f$ and $f_{i}, 0 \leqslant i \leqslant n-1$, where

$$
f_{i}\left(x, y, \ldots, y^{(n-1)}\right) \equiv \frac{\partial f}{\partial y^{(i)}}\left(x, y, \ldots, y^{(n-1)}\right)
$$

are continuous on $[a, b) \times R^{n}, a<b \leqslant \infty$.

(B) Solutions of (1) extend to $[a, b)$, and the compactness assumption.

(C) If $\left\{y_{j}(x)\right\}$ is a sequence of solutions of (1) which is uniformly bounded on a nondegenerate compact interval $[c, d] \subset[a, b)$, then there is a subsequence $\left\{y_{j_{k}}(x)\right\}$ such that $\left\{y_{j_{k}}^{(i)}(x)\right\}$ converges uniformly on each compact subinterval of $[a, b)$ for $i=0, \ldots, n-1$.

Let $y_{0}(x)$ be a solution of (1), then the linear differential equation

$$
z^{(n)}=\sum_{i=0}^{n-1} f_{i}\left(x, y_{0}(x), \ldots, y_{0}^{(n-1)}(x)\right) z^{(i)}
$$

is called [1] the variational equation of (1) along the solution $y_{0}(x)$.

Let $t \in[a, b)$. If there do not exist distinct solutions of (1) whose difference has $n$ zeros, counting multiplicities, on $[t, b)$ then we say (1) is disconjugate on $[t, b)$ and we signify this by writing $\eta_{1}(t)=b$. If (1) is not disconjugate on $[t, b)$, then the first conjugate point, $\eta_{1}(t)$, of $x=t$ for (1) is the infimum of the set of $t_{1} \in(t, b)$ such that there are distinct solutions of (1) whose difference has $n$ zeros, counting multiplicities, on $\left[t, t_{1}\right]$. For some results concerning $\eta_{1}(t)$ see [8] and [9]. We will denote the first conjugate point of $x=t$ for (2) by

Received by the editors December 15, 1975.

AMS (MOS) subject classifications (1970). Primary 34C10; Secondary 34B15.

Key words and phrases. Disconjugacy, boundary value problem, variational equation. 
$\eta_{1}\left(t ; y_{0}(x)\right)$. Our main result is that

$$
\eta_{1}(t)=\inf \left\{\eta_{1}\left(t ; y_{0}(x)\right): y_{0}(x) \text { is a solution of }(1)\right\}
$$

Henceforth the right-hand side of (3) will be denoted by $\inf _{\left(y_{0}(x)\right)} \eta_{1}\left(t ; y_{0}(x)\right)$. Spencer's main results in [9] were that if (A) and (B) hold, then $\eta_{1}(t)$ $\leqslant \inf _{\left(y_{0}(x)\right)} \eta_{1}\left(t ; y_{0}(x)\right)$ and noted that (3) holds when $n=2,3$. For $n=2$ (A) and (B) imply (C), and for $n=3$ if $\eta_{1}(t)>t$ on a dense subset of $[a, b)$, then (A) and (B) imply (C) (see [5]). To see that for $n=3$ (A) and (B) do not imply (C), see [4].

Let $i_{1}, \ldots, i_{k}$ be positive integers with $\sum_{j=1}^{k} i_{j}=n$; then the boundary value problem (BVP)(1),

$$
y^{\left(m_{j}\right)}\left(x_{j}\right)=A_{j_{m_{j}}}, \quad j=1, \ldots, k, 0 \leqslant m_{j} \leqslant i_{j}-1,
$$

is called an $\left(i_{1}, \ldots, i_{k}\right)$-BVP. Let $I$ be a subinterval of $[a, b)$, then we say that (1) is $\left(i_{1}, \ldots, i_{k}\right)$-disconjugate on $I$ provided for any $k$ points $x_{1}<\cdots<x_{k}$ in $I$ all $\left(i_{1}, \ldots, i_{k}\right)$-BVP's (1), (4) have at most one solution. Note that (1) is disconjugate on $I$ iff $(1)$ is $\left(i_{1}, \ldots, i_{k}\right)$-disconjugate on $I$ for all $k=2, \ldots, n$, $\sum_{j=1}^{k} i_{j}=n$.

THEOREM 1. If (A), (B) and (C) hold, then

$$
\eta_{1}(t)=\inf _{\left(y_{0}(x)\right)} \eta_{1}\left(t ; y_{0}(x)\right)
$$

Proof. Spencer [9] proved that $\eta_{1}(t) \leqslant \inf _{\left(y_{0}(x)\right)} \eta_{1}\left(t ; y_{0}(x)\right)$. To prove the theorem we will show that the assumption $\eta_{1}(t)<\inf _{\left(y_{0}\right)} \eta_{1}\left(t ; y_{0}(x)\right)$ leads to a contradiction. First we define an ordering (lexicographic ordering) for all $k$ tuples $\left(i_{1}, \ldots, i_{k}\right)$ with $\sum_{j=1}^{k} i_{j}=n, 2 \leqslant k \leqslant n$. We write $\left(j_{1}, \ldots, j_{l}\right)$ $>\left(i_{1}, \ldots, i_{k}\right)$ if $j_{1}>i_{1}$ or if there is an $s \in\{1, \ldots, l-1\}$ such that $j_{l}=i_{l}, l$ $=1, \ldots, s$, but $j_{s+1}>i_{s+1}$.

Since we are assuming that (1) is not disconjugate on $[t, \tau)$,

$$
\tau \equiv \inf _{\left(y_{0}\right)} \eta_{1}\left(t ; y_{0}(x)\right)
$$

there is at least one tuple $\left(j_{1}, \ldots, j_{l}\right)$ such that $(1)$ is not $\left(j_{1}, \ldots, j_{l}\right)$ disconjugate on $[t, \tau)$. Let $\left(i_{1}, \ldots, i_{k}\right)$ be the last of these tuples. By [9, Theorem 1.9], (1) is $(n-1,1)$-disconjugate on $[t, \tau)$ and so $\left(i_{1}, \ldots, i_{k}\right)<(n-1,1)$. Hence $i_{1}<n-1$. Since $\left(i_{1}+1, i_{2}, \ldots, i_{k}-1\right)>\left(i_{1}, \ldots, i_{k}\right)$ (if $i_{k}=1$, then by the $k$-tuple $\left(i_{1}+1, i_{2}, \ldots, i_{k}-1\right)$ we mean the $(k-1)$-tuple $\left(i_{1}+1, i_{2}, \ldots\right.$, $\left.\left.i_{k-1}\right)\right),(1)$ is $\left(i_{1}+1, i_{2}, \ldots, i_{k}-1\right)$-disconjugate on $[t, \tau)$.

Since $(1)$ is not $\left(i_{1}, \ldots, i_{k}\right)$-disconjugate on $[t, \tau)$, there are distinct solutions $y_{1}(x), y_{2}(x)$ of $(1)$ and $k$ points $t \leqslant x_{1}<x_{2}<\cdots<x_{k}<\tau$ such that

$$
y_{1}^{\left(m_{j}\right)}\left(x_{j}\right)=y_{2}^{\left(m_{j}\right)}\left(x_{j}\right)
$$


for $j=1, \ldots, k, m_{j}=0, \ldots, i_{j}-1$. Set $s_{1}=y_{1}^{\left(i_{1}\right)}\left(x_{1}\right)$ and $s_{2}=y_{2}^{\left(i_{1}\right)}\left(x_{1}\right)$. By the $\left(i_{1}+1, i_{2}, \ldots, i_{k}-1\right)$-disconjugacy, $s_{1} \neq s_{2}$.

For $s$ a real number consider the $\left(i_{1}+1, i_{2}, \ldots, i_{k}-1\right)$-BVP (1)

$$
\begin{aligned}
y^{\left(m_{j}\right)}\left(x_{j}\right) & =y_{1}^{\left(m_{j}\right)}\left(x_{j}\right), \quad j=1, \ldots, k-1, m_{j}=0, \ldots, i_{j}-1, \\
y^{\left(m_{1}\right)}\left(x_{1}\right) & =s, \\
y^{\left(m_{k}\right)}\left(x_{k}\right) & =y_{1}^{\left(m_{k}\right)}\left(x_{k}\right), \quad m_{k}=0, \ldots, i_{k}-2
\end{aligned}
$$

(if $i_{k}=1$ there is no boundary condition at $x_{k}$ ). By [10, Theorem 2.9], $S=\left\{s \in R\right.$ : the above $\left(i_{1}+1, i_{2}, \ldots, i_{k}-1\right)$-BVP has a solution $\}$ is an open interval (this is the place where the compactness assumption (C) is used in this proof). For each $s \in S$ let $y(x, s)$ be the solution of this BVP.

Now

$$
0=y_{1}^{\left(i_{k}-1\right)}\left(x_{k}\right)-y_{2}^{\left(i_{k}-1\right)}\left(x_{k}\right)=y^{\left(i_{k}-1\right)}\left(x_{k}, s_{1}\right)-y^{\left(i_{k}-1\right)}\left(x_{k}, s_{2}\right) .
$$

Since $S$ is connected we have from [6, Theorem 8] that

$$
0=\left(s_{1}-s_{2}\right) \frac{\partial y^{\left(i_{k}-1\right)}}{\partial s}\left(x_{k}, \bar{s}\right)=\left(s_{1}-s_{2}\right) z^{\left(i_{k}-1\right)}\left(x_{k}, \bar{s}\right)
$$

where $\bar{s}$ is between $s_{1}$ and $s_{2}$ and $z(x, \bar{s})=\partial y\left(x_{k}, \bar{s}\right) / \partial s$ is the solution of the $\left(i_{1}+1, i_{2}, \ldots, i_{k}-1\right)$-BVP

$$
\begin{aligned}
z^{(n)} & =\sum_{i=0}^{n-1} f_{i}\left(x, y(x, \bar{s}), \ldots, y^{(n-1)}(x, \bar{s})\right) z^{(i)}, \\
z^{\left(m_{j}\right)}\left(x_{j}\right) & =0, \quad j=1, \ldots, k-1, m_{j}=0, \ldots, i_{j}-1, \\
z^{\left(i_{1}\right)}\left(x_{1}\right) & =1, \\
z^{\left(m_{k}\right)}\left(x_{k}\right) & =0, \quad m_{k}=0, \ldots, i_{k}-2
\end{aligned}
$$

(if $i_{k}-1$ there is no boundary condition at $x_{k}$ ).

Since $s_{1} \neq s_{2}$ we have that $z^{\left(i_{k}-1\right)}\left(x_{k}, \bar{s}\right)=0$. This contradicts the $\left(i_{1}, i_{2}, \ldots\right.$, $i_{k}$ )-disconjugacy of (5) on $[t, \tau)$ and the proof is complete.

Hence Theorem 1 reduces disconjugacy of (1) to disconjugacy of corresponding linear differential equations. There are all kinds of sufficient conditions for disconjugacy of linear differential equations. Therefore using Theorem 1, all kinds of sufficient conditions for equation (1) to be disconjugate could be given. Instead of giving any of these nice results we will illustrate Theorem 1 by a new result for $n=4$. To this end consider the linear differential equations

$$
y^{(4)}=\sum_{i=0}^{3} p_{i}(x) y^{(i)},
$$




$$
y^{(4)}=\sum_{i=0}^{3} q_{i}(x) y^{(i)},
$$

where the coefficients are assumed to be continuous.

Let $u(x, \tau ; i), i=6,7$, be the solution of the initial value problem (i)

$$
y^{(j)}(\tau)=0, \quad j=0,1,2, \quad y^{\prime \prime \prime}(\tau)=1 .
$$

For results concerning when $u^{(j)}(x, \tau ; i)>0, j=0,1,2,3, x>\tau$ and $(-1)^{j} u^{(j)}(x, \tau ; i)<0, j=0,1,2,3, x<\tau$, see [3] and [7].

Theorem 2. Assume $n=4$ and (A), (B) and (C) hold. Assume $[c, d] \subset[a, b)$ and

$$
\begin{gathered}
p_{i}(x) \leqslant f_{i}\left(x, y, y^{\prime}, y^{\prime \prime}, y^{\prime \prime \prime}\right), \\
(-1)^{i} q_{i}(x) \leqslant(-1)^{i} f_{i}\left(x, y, y^{\prime}, y^{\prime \prime}, y^{\prime \prime \prime}\right),
\end{gathered}
$$

on $[c, d] \times R^{4}$ for $i=0,1,2,3$ and $f_{0}\left(x, y, y^{\prime}, y^{\prime \prime}, y^{\prime \prime \prime}\right) \leqslant 0$ on $[c, d] \times R^{4}$. If $u^{(j)}(x, d ; 6)>0$ on $(c, d]$ and $(-1)^{j} u^{(j)}(x, d ; 7)<0$ on $[c, d)$ for $j=0,1,2,3$, then $(1)$ is disconjugate on $[c, d]$.

PRoof. By Theorem 1 it suffices to show that all variational equations .

$$
z^{(4)}=\sum_{i=0}^{3} f_{i}\left(x, y_{0}(x), y_{0}^{\prime}(x), y_{0}^{\prime \prime}(x), y_{0}^{\prime \prime \prime}(x)\right) z^{(i)},
$$

where $y_{0}(x)$ is a solution of (1) with $n=4$, are disconjugate on $[c, d]$.

Since $u(x, c ; 6)$ is a solution of (6) satisfying

$$
u^{(j)}(x, c ; 6)>0 \quad \text { on }(c, d], \quad j=0,1,2,3,
$$

it follows easily that there is a solution $y_{3}(x)$ of (6) satisfying

$$
y_{3}^{(j)}(x)>0 \quad \text { on }[c, d], \quad j=0,1,2,3 .
$$

Similarly, since $-u(x, d ; 7)$ is a solution of (7) satisfying

$$
(-1)^{j} y^{(j)}(x)>0 \quad \text { on }[c, d), \quad j=0,1,2,3,
$$

there is a solution $y_{1}(x)$ of $(7)$ satisfying

$$
(-1)^{j} y_{1}^{(j)}(x)>0 \quad \text { on }[c, d], \quad j=0,1,2,3 .
$$

Set $y_{2}(x) \equiv 1$ on $[c, d]$. Then it is easy to show that the Wronskians $W\left[y_{1}, y_{2}\right], W\left[y_{1}, y_{3}\right], W\left[y_{2}, y_{3}\right]$, and $W\left[y_{1}, y_{2}, y_{3}\right]$ are positive on $[c, d]$. Define $L$ on $C^{4}[c, d]$ by

$$
L z(x)=z^{(4)}(x)-\sum_{j=0}^{3} f_{j}\left(x, y_{0}(x), y_{0}^{\prime}(x), y_{0}^{\prime \prime}(x), y_{0}^{\prime \prime \prime}(x)\right) z^{(j)}(x) .
$$

It is easy to check that 


$$
\begin{aligned}
& L y_{1}(x) \leqslant 0, \\
& L y_{2}(x)=-f_{0}\left(x, y_{0}(x), y_{0}^{\prime}(x), y_{0}^{\prime \prime}(x), y_{0}^{\prime \prime \prime}(x)\right) \geqslant 0, \\
& L y_{3}(x) \leqslant 0
\end{aligned}
$$

on $[c, d]$. Hence by [2, Proposition 4.1] $L z=0$ is disconjugate on $[c, d]$ and the proof is complete.

\section{REFERENCES}

1. P. Hartman, Ordinary differential equations, Wiley, New York, 1964. MR 30 \#1270.

2. - Principal solutions of disconjugate $n$-th order linear differential equations, Amer. J. Math. 91 (1969), 306-362. MR 40 \#450.

3. - On solutions of disconjugate differential equations, J. Math. Anal. Appl. 46 (1974), 338-351. MR 50 \#5094.

4. L. K. Jackson, A compactness condition for solutions of ordinary differential equations, Proc. Amer. Math. Soc. 57 (1976), 89-92.

5. L. K. Jackson and K. Schrader, Existence and uniqueness of solutions of boundary value problems for third order differential equations, J. Differential Equations 9 (1971), 46-54. MR 42 \#4813.

6. A. Peterson, Comparison theorems and existence theorems for ordinary differential equations, J. Math. Anal. Appl. (to appear).

7. - Existence-uniqueness for two-point boundary value problems for nth order nonlinear differential equations, Rocky Mountain J. Math. (submitted).

8. A. Peterson and D. Sukup, On the first conjugate point for nonlinear differential equations, Canad. Math. Bull. 18 (1975), 577-585.

9. J. Spencer, Relations between boundary value functions for a nonlinear differential equation and its variation equations, Canad. Math. Bull. 18 (1975), 269-276.

10. D. Sukup, On the existence of solutions to multipoint boundary value problems, Rocky Mountain J. Math. 6 (1976), 357-375.

Department of Mathematics, University of Nebraska, Lincoln, Nebraska 68588 